\title{
Rapid and strong human CD8+ T cell responses to vaccination with peptide, IFA, and CpG oligodeoxynucleotide 7909
}

\author{
Daniel E. Speiser, ${ }^{1}$ Danielle Liénard, ${ }^{1,2}$ Nathalie Rufer, ${ }^{3}$ Verena Rubio-Godoy, ${ }^{1}$ Donata Rimoldi, ${ }^{1}$ \\ Ferdy Lejeune, ${ }^{2}$ Arthur M. Krieg, ${ }^{4}$ Jean-Charles Cerottini, ${ }^{1,5}$ and Pedro Romero ${ }^{1}$

\begin{abstract}
1Division of Clinical Onco-Immunology, Ludwig Institute for Cancer Research, and 2Multidisciplinary Oncology Center, Centre Hospitalier Universitaire Vaudois (CHUV), Lausanne, Switzerland. ${ }^{3}$ Swiss Institute for Experimental Cancer Research, Epalinges, Switzerland. ${ }^{4}$ Coley Pharmaceutical Group,
\end{abstract} \\ Wellesley, Massachusetts, USA. 'Ludwig Institute for Cancer Research, Lausanne Branch, University of Lausanne, Epalinges, Switzerland.
}

\begin{abstract}
The induction of potent $\mathrm{CD8}^{+} \mathrm{T}$ cell responses by vaccines to fight microbes or tumors remains a major challenge, as many candidates for human vaccines have proved to be poorly immunogenic. Deoxycytidyl-deoxyguanosin oligodeoxynucleotides (CPG ODNs) trigger Toll-like receptor 9, resulting in dendritic cell maturation that can enhance immunogenicity of peptide-based vaccines in mice. We tested whether a synthetic ODN, CpG 7909 , could improve human tumor antigen-specific $\mathrm{CD8}^{+} \mathrm{T}$ cell responses. Eight HLA-A2 ${ }^{+}$melanoma patients received 4 monthly vaccinations of low-dose CPG 7909 mixed with melanoma antigen A (Melan-A; identical to MART-1) analog peptide and incomplete Freund's adjuvant. All patients exhibited rapid and strong antigenspecific $T$ cell responses: the frequency of Melan-A-specific $T$ cells reached over $3 \%$ of circulating $\mathrm{CD8}^{+} \mathrm{T}$ cells. This was one order of magnitude higher than the frequency seen in 8 control patients treated similarly but without CPG and 1-3 orders of magnitude higher than that seen in previous studies with synthetic vaccines. The enhanced $T$ cell populations consisted primarily of effector memory cells, which in part secreted IFN- $\gamma$ and expressed granzyme $B$ and perforin ex vivo. In vitro, $T$ cell clones recognized and killed melanoma cells in an antigen-specific manner. Thus, CpG 7909 is an efficient vaccine adjuvant that promotes strong antigenspecific $\mathrm{CD8}^{+} \mathrm{T}$ cell responses in humans.
\end{abstract}

\section{Introduction}

A major goal of therapeutic cancer vaccines is the induction of large numbers of antigen-specific $T$ cell populations with effector functions that are able to mediate immune protection. In contrast to viruses and other pathogens, vaccines containing recombinant proteins or synthetic antigenic peptides usually fail to induce significant immune responses unless they are mixed with adjuvants $(1,2)$. Effective adjuvants display at least 2 mechanisms of action: a depot effect that leads to prolonged antigen exposure in the host, and a capacity to trigger the innate immune system through activation of DCs via Toll-like receptors (TLRs) (3-5). Upon proper antigen presentation, activated DCs play a key role in the induction of T cell responses (6). Because of their high efficacy, several recently identified TLR ligands are promising vaccine adjuvants.

Synthetic deoxycytidyl-deoxyguanosin oligodeoxynucleotides (CpG ODNs) contain unmethylated CG motifs similar to those observed in bacterial DNA. CPG ODNs elicit a complex immunomodulatory cascade that includes the production of $\mathrm{T}$ helper-1type cells and proinflammatory cytokines (7). CpG ODNs directly stimulate DC activation through TLR9 triggering $(8,9)$, leading to enhanced $\mathrm{T}$ cell responses specific for coadministered antigens in mice (10-14). For example, we have reported previously that addition

Nonstandard abbreviations used: $\mathrm{CPG}$, deoxycytidyl-deoxyguanosin, IFA, incomplete Freund's adjuvant; Melan-A, melanoma antigen A; ODN, oligodeoxynucleotide; pDC, plasmacytoid DC; TLR, Toll-like receptor.

Conflict of interest: A.M. Krieg is an employee of Coley Pharmaceutical Group Inc. and owns stock in this company. The other authors have declared that no conflict of interest exists.

Citation for this article: J. Clin. Invest. 115:739-746 (2005)

doi:10.1172/JCI200523373. of CpG ODNs to melanoma antigen $\mathrm{A}_{26-35}$ peptide (Melan- $\mathrm{A}_{26-35}$ peptide; a widely used antigenic peptide in vaccine trials of HLA-A2 ${ }^{+}$ melanoma patients) mixed with incomplete Freund's adjuvant (IFA) increased Melan-A-specific T cell responses in HLA-A2 transgenic mice (15). However, the CPG motifs that stimulate the murine immune system are suboptimal for stimulating the human one. Indeed, TLR9-expressing human cells are susceptible to distinct CpG motifs (16-18). The recently described CpG 7909 has been optimized to stimulate human plasmacytoid DCs (pDCs) and B cells in vitro and in vivo (18).

Clinical studies have shown that CpG 7909 is a potent inducer of human innate immune responses and exhibits a strong adjuvant effect when coadministered with vaccines eliciting B cell responses against hepatitis B virus $(19,20)$. In contrast, it remains to be determined whether CPG ODNs are efficient adjuvants for vaccineinduced human cytolytic $\mathrm{T}$ cell responses (7). Given the well-documented but still relatively weak antigen-specific $\mathrm{CD}^{+} \mathrm{T}$ cell responses observed recently in melanoma patients vaccinated with Melan- $\mathrm{A}_{26-35}$ peptide and IFA (21-23), we tested whether coadministration of CPG 7909 to the same vaccine would enhance $\mathrm{T}$ cell responses. We have therefore performed a phase I clinical trial to examine toxicity and immunogenicity of this approach. Our results show rapid and consistent $\mathrm{T}$ cell responses in vivo, highlighting the potential of $\mathrm{CPG}$ 7909 to enhance cellular immune responses in humans.

\section{Results}

Vaccination with CpG 7909, peptide, and IFA caused no major side effects. Eight HLA-A2 ${ }^{+}$patients with advanced melanoma disease received 4 monthly subcutaneous injections of low doses of CpG 7909, Melan-A analog peptide, and IFA. Vaccination with this novel 


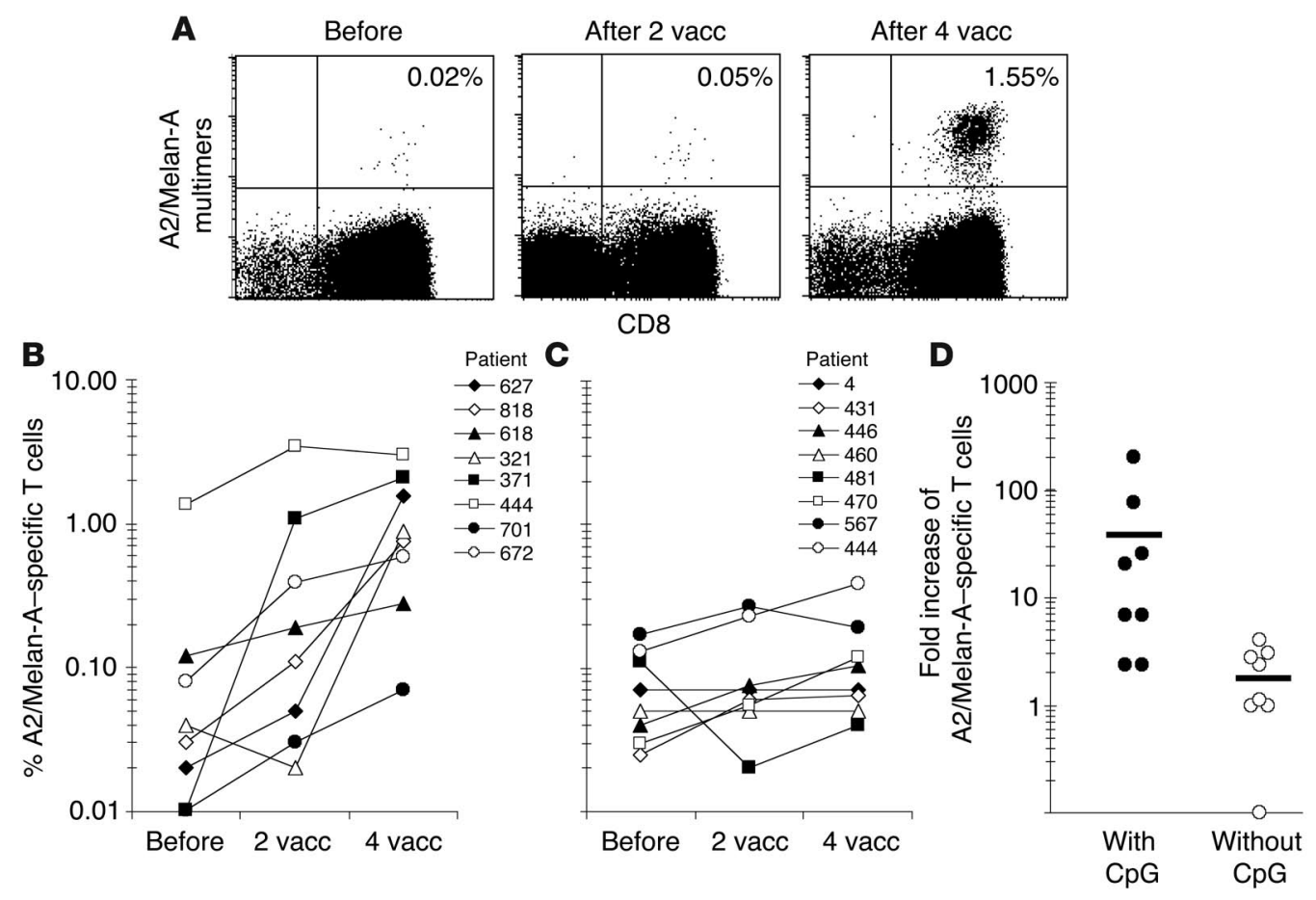

Figure 1

Rapid in vivo responses of Melan-A-specific T cells to vaccination (vacc) with low doses of CpG 7909, Melan-A peptide, and IFA. PBMCs were collected before, as well as 7-10 days after, vaccinations 2 and 4, and they were analyzed ex vivo by flow cytometry. (A) Dot plots from PBMCs of patient LAU 627, with percentage of Melan-A-specific cells among CD8 ${ }^{+}$T cells. (B) After 2 and 4 vaccinations, $6 / 8$ and $8 / 8$ patients, respectively, had significantly increased percentages (i.e., greater than 2-fold) of Melan-A-specific T cells. (C) A control group of 8 patients was similarly treated with Melan-A peptide and IFA but without CpG (23). After 2 vaccinations, none of the patients had more than 2-fold increased percentages. After 4 vaccinations, 4/8 patients had more than 2-fold increased frequencies, but percentages of Melan-A-specific $T$ cells remained significantly $(P<0.01)$ lower as compared to those of CpG-vaccinated patients. (D) Fold increase of Melan-A-specific T cells before or after 4 vaccinations in patients vaccinated with or without CpG. Horizontal lines indicate mean values.

3-component vaccine caused no major side effects. Minor systemic side effects were transient and included myalgia (4 patients), arthralgia and fatigue ( 3 patients), and nausea, malaise, and headache (2 patients). Interestingly, all 8 patients developed inflammatory signs at subcutaneous injection sites, with a peak of symptoms (induration, erythema, mild to moderate pain) around 2 weeks after injection. In response to recall vaccinations at distant sites in another limb, 4 patients showed reactivation of previous injection sites by redeveloping local inflammatory signs. Histological examination of a biopsy of one such distant reactivation site showed nonspecific inflammation with predominant perivascular lymphocyte infiltration (data not shown).

Rapid and consistent in vivo generation of Melan-A-specific $C D 8^{+} T$ cells. PBMCs collected before and after vaccination were analyzed ex vivo by flow cytometry using fluorescent CD8-specific antibody and HLA-A2/Melan-A peptide multimers. After 4 vaccinations with CpG 7909, Melan-A peptide, and IFA, all 8 patients exhibited increased frequencies of Melan-A-specific CD8 ${ }^{+} \mathrm{T}$ cells $(0.07-3.00 \%)$, resulting in significantly $(P<0.01)$ higher percentages than before vaccination (Figure $1, \mathrm{~A}$ and $\mathrm{B}$ ).

After 4 monthly vaccinations, the 8 patients exhibited a frequency of $1.15 \% \pm 0.93 \%$ (mean \pm SD) Melan-A-specific $\mathrm{CD}^{+} \mathrm{T}$ cells. A control group of 8 melanoma patients was treated similarly but without $\mathrm{CpG}$ (Figure $1 \mathrm{C}$ ), resulting in significantly $(P<0.01)$ lower $\mathrm{T}$ cell frequencies $(0.13 \% \pm 0.11 \%)$. Also, the response rate was far below $100 \%$, since only 4 out of 8 patients generated ex vivo detectable $\mathrm{T}$ cell responses after 4 vaccinations. $\mathrm{CpG}$-vaccinated patients reached a mean of 43 -fold higher Melan-A-specific $\mathrm{CD}^{+} \mathrm{T}$ cell frequencies than before vaccination, whereas patients vaccinated without $\mathrm{C}$ PG reached a mean of 1.9-fold higher frequencies than before vaccination (Figure 1D).

The majority of T cell responses developed rapidly: 6 out of 8 patients had increased frequencies of Melan-A-specific $\mathrm{CD}^{+} \mathrm{T}$ cells after just 2 vaccinations (Figure 1B). This is again in sharp contrast to previous results. In the control group (Figure 1C), the lack of early responses (after 2 vaccinations) and the relatively low response rate are characteristic of all other studies with low-dose synthetic vaccines (21-25). Interestingly, patient LAU 444, who already had high levels of Melan-A-specific CD8 ${ }^{+} \mathrm{T}$ cells before vaccination (resulting from previous immunotherapy and a natural response to melanoma [26]), was the only patient whose frequency peaked after just 2 vaccinations. In the other 7 patients, maximal frequencies were reached after 4 vaccinations. To our knowledge, these results demonstrate for the first time in humans that a synthetic peptide-based vaccine, when coadministered with adequate adjuvant, can rapidly elicit ex vivo detectable $\mathrm{CD}^{+} \mathrm{T}$ cell responses.

Two of the 8 patients (LAU 371 and LAU 321) had previously been unsuccessfully vaccinated with Melan-A peptide mixed with the immunological adjuvants MPL and QS21 (Figure 2). During 4-5 years, the frequencies of Melan-A-specific T cells 


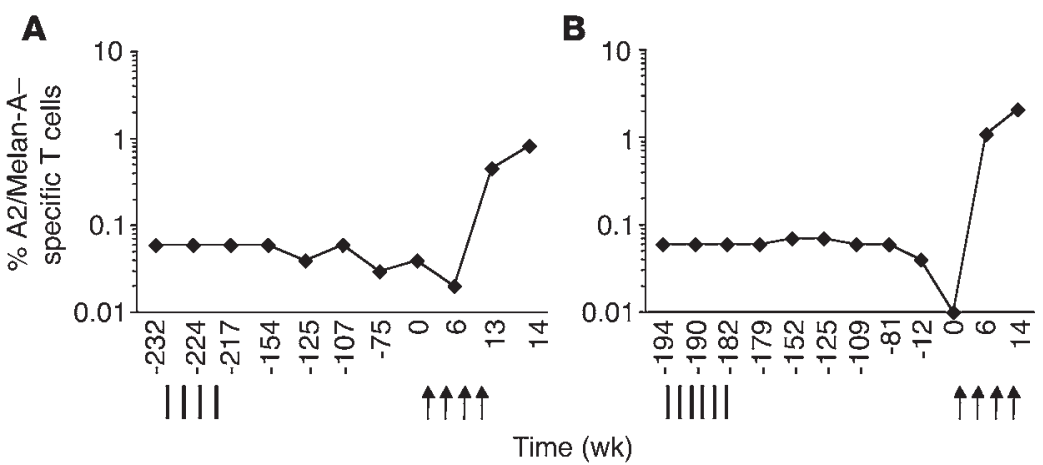

Figure 2

$\mathrm{T}$ cell responses to $\mathrm{CpG}$ vaccination (arrows) in patients that previously did not respond to vaccination with Melan-A peptide and immunological adjuvants MPL and QS21 (vertical lines). Percentages of multimer ${ }^{+} T$ cells were determined ex vivo in PBMCs collected over an observation period of 4 to 5 years. (A) Patient LAU 321; (B) Patient LAU 371.

remained at stable low values (patient LAU 321, $0.05 \% \pm 0.01 \%$; patient LAU $371,0.06 \% \pm 0.02 \%$ ), a frequent observation in immune nonresponders (27). Nevertheless, both patients responded rapidly and strongly to vaccination with CpG 7909, Melan-A peptide, and IFA (Figure 2).

$T$ cell frequencies peaked 7-10 days after recall vaccination. Results in Figures 1 and 2 were obtained with blood samples collected 7-10 days following the monthly recall vaccinations (i.e., vaccinations 2 and 4). To determine the short-term kinetics of T cell responses, we analyzed additional blood samples collected immediately before and 14 days after recall vaccination with CpG 7909, Melan-A peptide, and IFA. The highest $\mathrm{T}$ cell levels were reached 7-10 days after recall vaccination (Figure 3). Maximal frequencies (as shown in Figures 1 and 2) were observed on day 7 in 4 patients (LAU 818, LAU 618, LAU 701, and LAU 672) and on day 10 in 3 patients (LAU 627, LAU 371, and LAU 444). Interestingly, frequencies of circulating Melan-A-specific T cells dropped to relatively low levels 14 days after recall vaccination (frequencies for patient LAU 321 were not evaluable, since blood samples from days 7 and 14 were not available). Thus, recall vaccination with CPG 7909, Melan-A peptide, and IFA led to increased $T$ cell frequencies during the first 7-10 days, followed by a decline shortly afterwards, similarly to previous observations in mice (28-30).

Predominance of effector memory $T$ cells expressing effector genes in vivo. It is well established that Melan-A-specific T cells in melanoma patients comprise naive and activated cells (31). To determine the differentiation state of vaccine-induced Melan-A-specific T cells, we first assessed CD45RA and CCR7 cell surface expression $(32,33)$ by Melan-A-specific T cells. Before vaccination, the majority were naive $\mathrm{CCR} 7^{+} \mathrm{CD} 45 \mathrm{RA}^{+} \mathrm{T}$ cells (Figure 4A). In contrast, after vaccination most Melan-A-specific $\mathrm{T}$ cells displayed an effector memory phenotype (CD45RA-CCR7-; Figure 4A and Table 1). After 4 vaccinations, effector memory cells accounted for $82 \% \pm 13 \%$ of Melan-A multimer ${ }^{+} \mathrm{T}$ cells (mean $\pm \mathrm{SD}$ of the 8 patients), and naive, central memory, and effector $\mathrm{T}$ cells were only found at low percentages. To assess effector gene expression directly, we sorted Melan-A-specific T cell subpopulations according to their expression of CD45RA and CCR7. As described previously (33), we sorted 5-cell aliquots and isolated mRNA, which was transcribed to cDNA then nonspecifically amplified and finally used for PCR with sequence-specific primers. All cells were positive for CD3 (Figure 4B). As expected, naive cells, which are not cytolytic and do not produce cytokines, did not contain detectable granzyme B, perforin, TNF- $\alpha$, or NK receptor CD94 mRNA, and they only rarely gave an IFN- $\gamma$ signal. After vaccination, granzyme B, perforin, and IFN- $\gamma$ mRNA transcripts were found in significant fractions of 5-cell aliquots of effector memory cells. Finally, after 4 vaccinations, effector memory cells showed increased expression of granzyme B and perforin, and some fractions of 5-cell aliquots also expressed TNF- $\gamma$ and NK receptor CD94.

Activated $T$ cells with fine specificity to the natural tumor antigen. All vaccinations were done with the Melan-A analog peptide ELAGIGILTV, which contains the amino acid leucine in position 2 instead of the natural amino acid alanine. This substitution causes the analog peptide to bind more strongly and stably to HLA-A2, resulting in increased antigenicity and immunogenicity (31). To test the ability of $\mathrm{T}$ cells elicited by the vaccine to recognize A2/Melan-A antigen, we assessed the fine specificity of Melan-A-specific $\mathrm{T}$ cells in IFN- $\gamma$ Elispot assays performed ex vivo. After vaccination, Melan-A-specific, IFN- $\gamma$-producing $\mathrm{T}$ cells from all 8 patients reached frequencies above the detection limit of $0.01 \%$ of $\mathrm{CD}^{+}$ T cells (Figure $5 \mathrm{~A}$ and Table 2). The natural and analog peptides triggered comparable frequencies of Elispot-forming cells $(0.12 \% \pm 0.12 \%$ and $0.14 \% \pm 0.10 \%$, respectively). To assess the functional avidity, we tested the cytotoxic activity of A2/Melan-Aspecific $\mathrm{T}$ cell clones derived from patients after vaccination. Cytotoxicity was peptide-specific and occurred at low peptide concentrations (Figure 5B), demonstrating high functional avidity to A2/Melan-A. The observed hierarchy of peptide recognition efficiency is characteristic for the majority of Melan-Aspecific $\mathrm{T}$ cell clones and is in accordance with the hierarchy of binding strength of the 3 peptide variants to HLA-A*0201 (31). In agreement with these findings, the clones indeed killed the $\mathrm{A} 2^{+} /$Melan- $\mathrm{A}^{+}$melanoma cell line Me 275, but not the $\mathrm{A}^{+} /$Melan-A- melanoma cell line NA8 (Figure 5C). These data

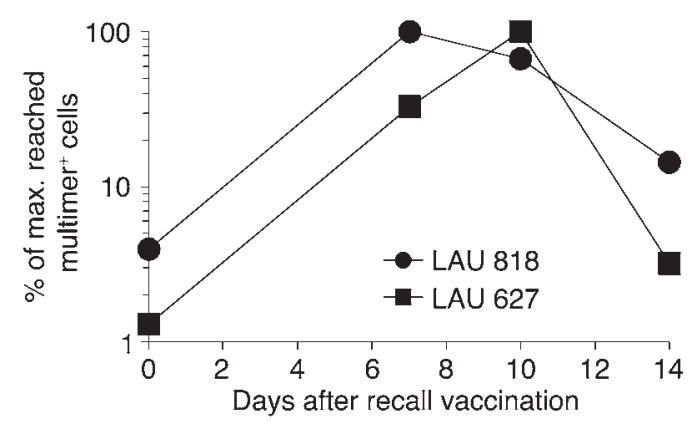

\section{Figure 3}

Short-term kinetics of Melan-A-specific T cells from 2 representative patients with maximal (max.) responses on day 7 (patient LAU 818) and on day 10 (patient LAU 627) after recall vaccination with $\mathrm{CpG}$ 7909, Melan-A peptide, and IFA. PBMCs were collected immediately before, as well as 7,10 , and 14 days after, recall vaccination. Percentages of multimer ${ }^{+} \mathrm{T}$ cells were determined ex vivo and calculated in percentages of maximally reached values $(100 \%)$ per patient. 
A

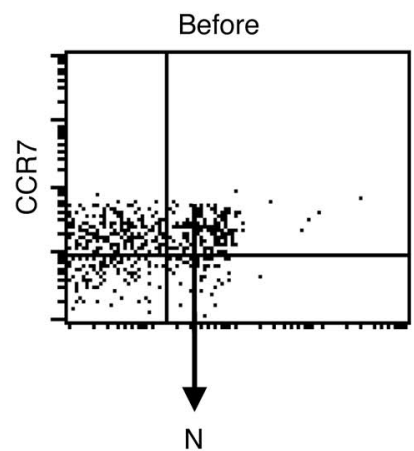

B

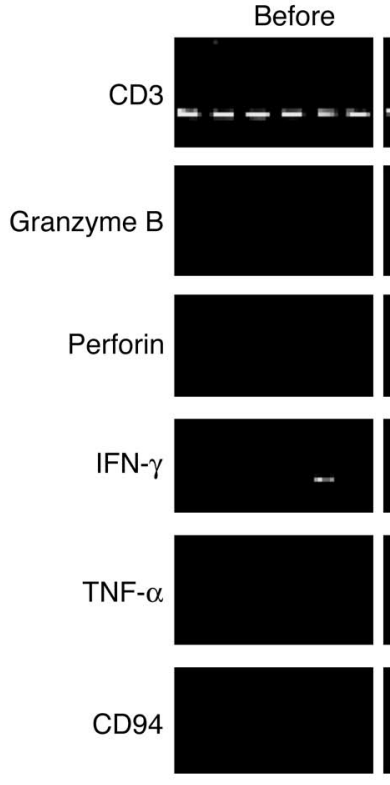

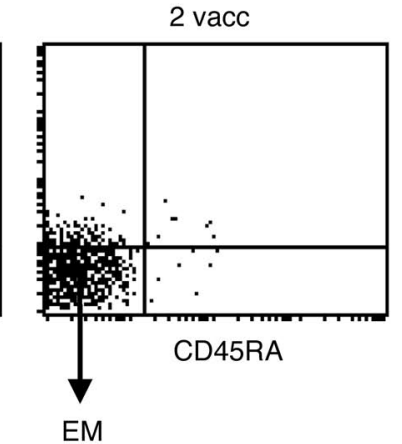

EM

2 vacc
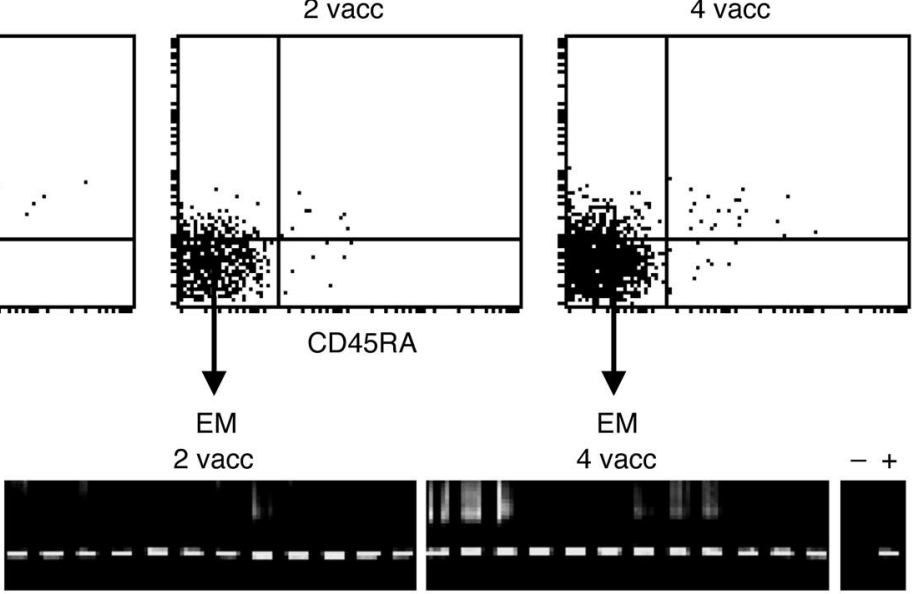

EM
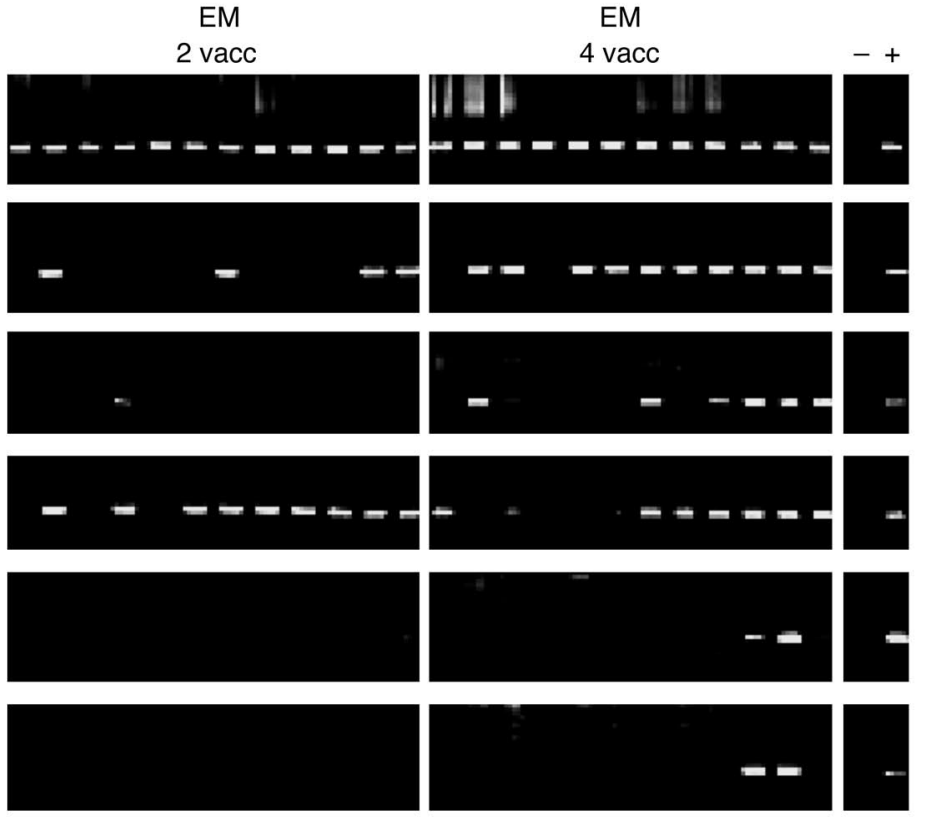

\section{Figure 4}

Expression of effector mediators by vaccine-activated MelanA-specific T cells. Multiparameter cytometric analysis and sorting was performed with A2/Melan-A multimers and antibodies specific for CD8, CD45RA, and CCR7. (A) Expression of CD45RA and CCR7 is shown for A2/Melan-A multimer ${ }^{+} \mathrm{CD} 8^{+}$gated cells. (B) RT-PCR gene expression analysis was performed (33) using primers specific for $C D 3$, granzyme $B$, perforin, IFN- $\gamma$, TNF- $\alpha$, and CD94, on sorted A2/Melan-A multimer ${ }^{+}$ $T$ cells which were $R A{ }^{+} \mathrm{CCR} 7{ }^{+}$ (naive cells) or RA-CCR7+ effector memory cells. Each band represents the RT-PCR product from RNA isolated from sorted 5-cell aliquots. Data in A and B (representative of 8 and 2 patients analyzed, respectively) are from PBMCs collected from patient LAU 371 before and after 2 and 4 vaccinations. +, positive control; -, negative control; $\mathrm{N}$, naive $\mathrm{T}$ cells; EM, effector memory T cells. demonstrate that $\mathrm{T}$ cells induced by a vaccine comprised of a synthetic Melan-A analog peptide specifically recognized the natural Melan-A antigen expressed by tumor cells.

\section{Discussion}

This study represents the first human trial of CPG ODN combined with a $\mathrm{T}$ cell peptide antigen. It demonstrates that $\mathrm{CpG}$ 7909 is an efficient adjuvant that promotes rapid antigen-specific $\mathrm{CD}^{+} \mathrm{T}$ cell responses. As compared to vaccination without $\mathrm{CpG}$, responses were 1 order of magnitude higher. Synthetic peptide plus adjuvant vaccines have been shown to induce only low frequencies of circulating antigen-specific $T$ cells, despite administration of 8 or more injections $(34,35)$. Moreover, in the majority of cancer patient studies reported so far, $\mathrm{T}$ cell responses could not be detected ex vivo but rather only after 1 or more rounds of in vitro $T$ cell stimulation and proliferation $(21,24,36,37)$. A small number of studies (using $0.5-1 \mathrm{mg}$ peptide per vaccination plus adjuvants or cytokines) described ex vivo detectable $\mathrm{T}$ cell responses $(22,25)$, but $\mathrm{T}$ cell frequencies were still 10-100 times lower than what we observed in the present study. Only 1 study reported higher percentages of antigen-specific T cells following vaccinations with the particularly immunogenic gp100 T2M analog peptide and IFA. However, very high doses were used for vaccination: the cumulative peptide dose was 100 -fold higher and the number of vaccinations 10-fold higher than in our study (38).
The HLA-A2/Melan-A antigenic system constitutes a welldefined model for studies of spontaneous and vaccine-induced $\mathrm{CD}^{+} \mathrm{T}$ cell responses in humans $(31,37)$. Besides the gp100 analog peptide 2TM, Melan-A peptide is one of the rare cancer peptide antigens capable of inducing ex vivo detectable $\mathrm{T}$ cell responses in relatively large proportions of patients $(22,37)$. The availability of strong adjuvants such as CpG 7909 makes it possible to investigate the immunogenicity of weaker antigens, which make up the majority of known cancer epitopes.

Both CpG and peptide were given at low doses. Future studies will test whether increased doses of CPG 7909 and/or peptides can further enhance $T$ cell activation. CPG 7909 can be administered to humans with generally acceptable tolerability at doses up to at least $20 \mathrm{mg}$ per injection weekly for 6 months or longer (A. Krieg, unpublished observations), that is, at cumulative doses that are 250 -fold higher than in our study. Very high CPG ODN doses $(2.5 \mathrm{mg} / \mathrm{kg})$ given daily for 20 days have recently been reported to destroy lymphoid tissue structures in mice (39). High-dose effects also include extramedullary hematopoeisis and a lethal systemic inflammatory response syndrome in mice (40). The potential toxicities of highdose CpG 7909 administration in humans are likely to be largely different from those reported in mice, due to the much more restricted distribution of TLR9 expression in human as compared to mouse immune cells $(5,17)$. Our patients had no apparent liver toxicity, no enlargement of lymph nodes or spleen, and intact antigen-specific B 
Table 1

Percentages of naive and non-naive Melan-A-specific T cells

\begin{tabular}{lcccc}
\hline & & & & \\
CD45RA & $\mathrm{T}_{\mathrm{N}}$ & $\mathrm{T}_{\mathrm{CM}}$ & $\mathrm{T}_{\mathrm{EM}}$ & $\mathrm{T}_{\mathrm{E}}$ \\
CCR7 & + & - & - & + \\
LAU 627 & + & + & - & - \\
LAU 818 & 0 & 4 & 93 & 2 \\
LAU 618 & 0.7 & 7 & 89 & 3 \\
LAU 321 & 0.1 & 8 & 91 & 0.8 \\
LAU 371 & 5 & 30 & 60 & 5 \\
LAU 444 & 1 & 15 & 84 & 0.6 \\
LAU 701 & 2 & 1 & 93 & 5 \\
LAU 672 & 5 & 5 & 62 & 31 \\
Mean & 2 & 7 & 86 & 3 \\
SD & 2 & 9 & 82 & 6 \\
& & & 10 & 9 \\
\hline
\end{tabular}

Percentages of naive $\left(T_{N} ; R A+C C R 7^{+}\right)$, central memory $\left(T_{C M}\right.$; $\mathrm{RA}^{-} \mathrm{CCR} 7^{+}$), effector memory ( $\left.\mathrm{T}_{\mathrm{EM}} ; \mathrm{RA}^{-} \mathrm{CCR} 7^{-}\right)$, and effector ( $\mathrm{T}_{\mathrm{E}}$;

$\left.\mathrm{RA}^{+} \mathrm{CCR} 7^{-}\right) \mathrm{T}$ cells were determined among A2/Melan-A multimer+ gated T cells from PBMCs collected $7-10$ days after the fourth vaccination.

cell responses (data not shown). Two lymph node biopsies after CpG 7909 vaccination showed follicles with normal structure. Interestingly, 4 of 8 patients developed circulating anti-dsDNA antibodies as detected by ELISA, but there were no clinical signs for autoimmune disease, and systemic inflammatory markers in sera (IP-10, $\mathrm{CRP}$ ) remained normal. We are currently investigating the possibility that the antibodies could be specific for CPG 7909 .

Out of the 8 patients included in the study, 3 had no evidence for disease at study entry; during the study, 1 of them remained disease-free, 1 progressed, and 1 showed local tumor relapse. Among the 5 patients with measurable disease at study entry, 1 had stable disease, 2 progressed, and 2 showed local relapse. This phase I study was not designed to assess tumor response, and the follow-up time was short (mean of $7 \pm 2$ months after inclusion in the study). Comprehensive clinical results need to be obtained in phase II trials with more patients and a longer follow-up time.
In contrast to mouse TLR9, human TLR9 is only expressed by pDCs and B cells. Presumably, B cells are not involved in T cell activation, suggesting that $\mathrm{PDCs}$ were responsible for the observed $\mathrm{T}$ cell responses (41). One important question is whether our vaccine was capable of priming $\mathrm{T}$ cells, or whether the observed responses were due to reactivation and/or redistribution of previously primed cells. Remarkably, the levels of circulating naive Melan-A-specific T cells were abnormally low after CPG 7909 vaccination (undetectable in 4 patients, $0.03 \% \pm 0.02 \%$ in the remaining 4 patients). For comparison, naive Melan-A-specific T cells are present at relatively high frequencies $(0.07 \% \pm 0.06 \%)$ in untreated healthy individuals and melanoma patients (42). Our observations that all patients responded, and that naive cell frequencies were low after vaccination, suggest that CPG 7909 vaccination was indeed capable of priming naive $\mathrm{T}$ cells. This conclusion is also supported by recent mouse experiments showing that $\mathrm{CpG}$ ODN-matured pDCs can prime $\mathrm{CD}^{+} \mathrm{T}$ cells in vivo (41).

We also analyzed whether the prevaccination immune status differed between patients treated with and without CpG. However, we did not find significant differences with regard to prevaccination frequencies of naive cells or of each of the 3 other Melan-A-specific T cell populations defined by CD45RA/CCR7 expression. Data were interpretable from 3 patients of each group (data not shown). We have recently demonstrated that the percentage of preexisting CD28 negative Melan-A-specific $T$ cells correlates with $T$ cell responsiveness to vaccination with peptide and IFA in stage III-IV melanoma patients (26). Analyzing this aspect in the present study, we could not find a statistically significant difference between the 2 patient groups (mean prevaccination percentages \pm SD of CD28 negative Melan-A-specific T cells were $28 \% \pm 33 \%$ and $29 \% \pm 27 \%$ in patients treated with and without $\mathrm{CpG}$, respectively). Data were interpretable from 6 patients of each group (data not shown). Based on these findings, one may speculate that vaccination with $\mathrm{CpG}$ induces increased frequencies of circulating $T$ cells independently of the patient's prevaccination immune status, whereas $T$ cell responses to peptide and IFA vaccination (without $\mathrm{CpG}$ ) depend significantly on endogenous (tumor-driven) immunogenicity (26).

\section{Figure 5}

$T$ cell receptor fine-specificity and tumor cell recognition. (A) PBMCs from patient LAU 371 were tested ex vivo in IFN- $\gamma$ Elispot assays before and after vaccination with HIV, tyrosinase, and Melan-A natural and analog peptides. (B) Melan-A-specific $T$ cells were sorted by flow cytometry and cloned, and cytotoxicity was tested against T2 cells in the presence of the following titrated HLA-A2 binding peptides: Melan-A analog (squares), Melan-A natural decamer (circles), Melan-A natural nonamer (triangles), and influenza matrix protein GILGFVFTL (diamonds). (C) Cytotoxicity against $\mathrm{HLA}-\mathrm{A2}^{+}$melanoma cell lines Me 275 (Melan- $A^{+}$; filled symbols) and NA8 (Melan-A-; open symbols) in the presence (squares) or absence (circles) of synthetic Melan-A analog peptide. Data shown were generated with clone 6 derived from patient LAU 371 and are representative for 12 of 22 clones generated from patients LAU 371 and LAU 444.

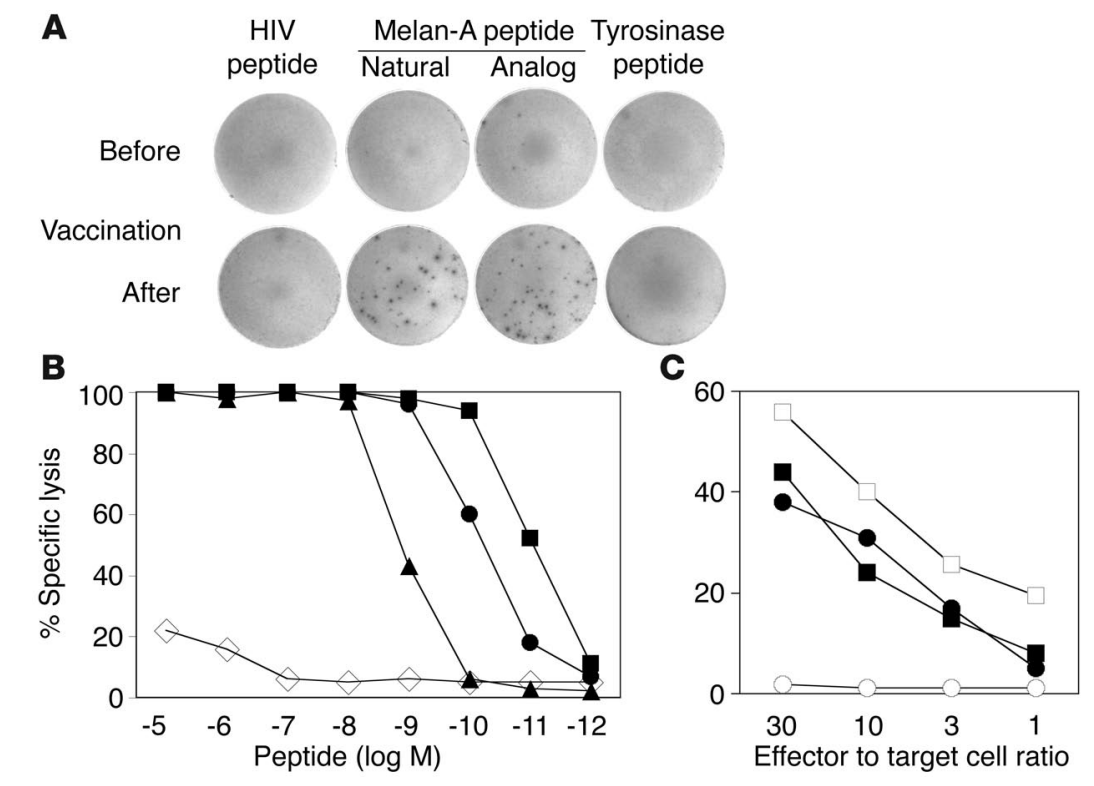




\section{Table 2}

Percentage of IFN- $\gamma$ Elispot-forming cells

\begin{tabular}{lcc} 
& \multicolumn{2}{c}{ Melan-A peptide } \\
Patient & Natural & Analog \\
LAU 627 & 0.08 & 0.08 \\
LAU 818 & 0.05 & 0.06 \\
LAU 618 & 0.07 & 0.13 \\
LAU 321 & 0.02 & $\leq 0.01$ \\
LAU 371 & 0.11 & 0.12 \\
LAU 444 & 0.40 & 0.32 \\
LAU 701 & 0.02 & 0.02 \\
LAU 672 & 0.19 & 0.23 \\
Mean & 0.12 & 0.14 \\
SD & 0.12 & 0.10 \\
\hline
\end{tabular}

Percentages of Elispot-forming cells among CD8 ${ }^{+} \mathrm{T}$ cells in PBMCs are shown for all 8 patients after vaccination. Background results with HIV polymerase peptide and without peptide were below the detection limit of $0.01 \%$ (not shown).

The pattern of specific $\mathrm{T}$ cell expansion and contraction following recall vaccination is reminiscent of acute $\mathrm{T}$ cell responses observed in mice (28-30). Interestingly, in patients vaccinated with Melan-A and IFA without CpG, such rapid changes of specific $T$ cell frequencies were not observed (data not shown). In the majority of cancer vaccine studies reported so far, antigen-specific $\mathrm{T}$ cells were analyzed at only a single time point after each vaccination. With the introduction of highly efficient adjuvants such as CPG 7909, stronger, shorter-lasting $\mathrm{T}$ cell responses can be observed. Thus, antigen-specific $T$ cells should be monitored at multiple time points after vaccination.

One possible explanation for the rapid decline during the second week after recall vaccination is $\mathrm{T}$ cell migration into lymphoid and tumor tissues (43). We were able to investigate metastases surgically removed from 3 patients after vaccination with CpG 7909, Melan-A, and IFA, and found that $5.03 \%, 0.12 \%$, and $0.08 \%$ of $\mathrm{CD}^{+} \mathrm{T}$ cells were specific for Melan-A (data not shown). Ongoing studies will address the question of whether vaccine-activated $T$ cell clones are found in these lesions. We are also developing techniques to estimate the percentage of $T$ cells that undergo apoptosis and/or migrate to other compartments $(29,44)$.

The vaccine-induced $T$ cell populations consisted predominantly of effector memory $\mathrm{T}$ cells (CD45RA-CCR7-). Central memory and effector cells were also induced, but to a much lesser extent. Most non-naive cells expressed IFN- $\gamma$ mRNA. Effector memory cells expressed granzyme B mRNA, and some of them expressed perforin mRNA. While this was the case for the majority of 5-cell aliquots after 4 vaccinations, only a few cells were positive for granzyme B after only 2 vaccinations. Finally, TNF- $\alpha$ and CD94 were expressed by only a small fraction of cells. Through our ongoing studies, we aim to elucidate whether multiple recall vaccinations can induce effector functions in the majority of cells.

It is conceivable that the magnitude and durability of antigenspecific $\mathrm{T}$ cell responses induced by peptide-based vaccines could be further enhanced by the use of other types of CpG ODNs, such as the $\mathrm{C}$ type, which recently has been reported to induce higher levels of IFN- $\alpha$ secretion than the B type, which was used in this study (45-48). Indeed, IFN- $\alpha$ appears to improve the development of $\mathrm{T}$ cell memory $(49,50)$. Moreover, various other
TLR ligands are becoming available for use in humans and are interesting candidates as adjuvants for vaccination and induction of protective immunity.

In conclusion, this study underscores the synergy of combined triggering of innate and specific immunity. The TLR9 ligand CpG 7909 is a potent stimulator of innate immune cells, and its coadministration with antigen induces high numbers of antigen-specific $\mathrm{CD}^{+} \mathrm{T}$ cells. Our findings suggest that further approaches to $\mathrm{T}$ cell-directed immunotherapy should take advantage of such a synergy.

\section{Methods}

Patients, eligibility criteria, and study protocol. HLA-A2 ${ }^{+}$patients with histologically proven metastatic melanoma of the skin expressing Melan-A/MART-1 (determined by RT-PCR or immunohistochemistry) were included after informed consent was obtained in this phase I prospective trial of the Ludwig Institute for Cancer Research (LICR). Study protocols were approved by the LICR protocol review committee, as well as by the medical and ethical committees of the University Hospital (Lausanne, Switzerland). Inclusion criteria were as follows: Karnofsky performance status of at least $70 \%$, normal complete blood count and kidney-liver function, and no concomitant anti-tumor therapy or immunosuppressive drugs. Exclusion criteria were pregnancy, seropositivity for HIV-1 Ab or hepatitis B surface antigen, brain metastasis, uncontrolled bleeding, clinically significant autoimmune disease, or New York Heart Association class III-IV heart disease. Study end points were toxicity and $\mathrm{CD}^{+} \mathrm{T}$ cell response. All patients were evaluated for immune response and treatment toxicity. Patients received 4 vaccinations subcutaneously in monthly intervals. The low-dose vaccines were composed of $500 \mu \mathrm{g}$ CpG 7909, $100 \mu \mathrm{g}$ Melan-A analog peptide, and $300 \mu$ IFA (Montanide ISA-51; Seppic) all mixed together and prepared in a syringe as a stable emulsion. Phosphorothioate backbone CpG 7909 (TCGTCGTTTTGTCGTTTTGTCGTT) was produced by Coley Pharmaceutical Group under good manufacturing practice conditions, and had no detectable endotoxin by limulus amebocyte lysate assay. The Melan-A analog peptide $_{26-35}$ ELAGIGILTV was synthesized by NeoMPS Inc. and formulated $(330 \mu \mathrm{g} / \mathrm{ml}$ in PBS $/ 30 \%$ DMSO) by the Biological Production Facility, LICR Melbourne. The IFA (Montanide ISA-51; Seppic) contained mineral oil (Drakeol) and anhydro mannitol octadecanoate. Control patients were treated similarly with 4 monthly vaccinations as described previously (23), except the vaccines did not contain CPG ODN. As in most phase I studies, patients were not randomized, but first assigned to the control group and subsequently to the CPG group. Patient LAU 444 was first included in the control group, and 2 years later in the $\mathrm{CpG}$ group.

Blood cells, HLA-A2/peptide multimers, flow cytometry, and IFN- $\gamma$ Elispot assays. Ficoll-Paque centrifuged PBMCs $\left(1 \times 10^{7}-2 \times 10^{7}\right)$ were cryopreserved in RPMI 1640, 40\% FCS, and 10\% DMSO. Phycoerythrin-labeled HLA-A*0201/peptide multimers (originally called tetramers) were prepared as described previously $(42,51)$ with Melan-A analog peptide $26-35$ ELAGIGILTV. Anti-CD8, anti-CD28, and allophycocyanin-conjugated goat anti-rat IgG were purchased from BD Biosciences and anti-CD45RA was from Immunotech. Anti-CCR7 rat IgG2a mAb 3D12 was provided by Martin Lipp (Max Delbrueck Center for Molecular Medicine, Berlin, Germany). Five color stains were done with HLA-A2/peptide multimers, FITCconjugated anti-CD28, PE-Texas Red-conjugated anti-CD45RA, allophycocyanin-Cy7-conjugated anti-CD8 reagents, and anti- 
CCR7 mAb followed by allophycocyanin-conjugated goat anti-rat IgG antibody. Briefly, CD8 ${ }^{+} \mathrm{T}$ cells were enriched using a MiniMACS device (Miltenyi Biotec) resulting in more than $90 \% \mathrm{CD}^{+} \mathrm{CD}^{+}$cells. Cells $\left(10^{6}\right)$ were incubated with multimers $(1 \mu \mathrm{g} / \mathrm{ml}, 60$ minutes, room temperature) and then with antibodies $\left(30\right.$ minutes, $\left.4^{\circ} \mathrm{C}\right)$. We acquired $5 \times 10^{5} \mathrm{CD}^{+} \mathrm{T}$ cells per sample with a FACSVantage machine, and data were analyzed with CellQuest software (BD Biosciences). IFN- $\gamma$ Elispot assays were performed using IFN- $\gamma$-specific antibodies (Diaclone, Biotest). Briefly, plates were coated overnight with antibody to human IFN- $\gamma$ and washed 6 times. We added $1.66 \times 10^{5} \mathrm{PBMCs} /$ well in $200 \mu \mathrm{l}$ Iscove medium (Gibco; Invitrogen Corp.) supplemented with $8 \%$ human serum and $10 \mu \mathrm{g} / \mathrm{ml}$ peptide and incubated it for 16 hours at $37^{\circ} \mathrm{C}$. Assays were performed in 6 replicates, without peptide or with peptides derived from Melan-A (natural EAAGIGILTV, analog ELAGIGILTV), tyrosinase YMDGTMSQV, and HIV-1 polymerase ILKEPVHGV. Cells were removed, and plates were developed with a second (biotinylated) antibody to human IFN- $\gamma$ and streptavidin-alkaline phosphatase (Diaclone, Biotest). The spots were revealed with BCIP/NBT substrate (Sigma Tablets; Sigma-Aldrich) and counted with an automatic reader (Bioreader 2000; BioSys $\mathrm{GmbH}$ ). The percentage of $\mathrm{CD}^{+} \mathrm{CD}^{+} \mathrm{PBMCs}$ was determined by flow cytometry on the same batch of cryopreserved cells. Results of both multimer ${ }^{+} \mathrm{T}$ cells and Elispot-forming $\mathrm{T}$ cells were calculated as a percentage of $\mathrm{CD} 8^{+} \mathrm{T}$ cells. For each patient and assay system, pre- and post-vaccination samples were thawed and tested in the same experiment.

Quality control of laboratory immune monitoring. Standardization of multimer and IFN- $\gamma$ Elispot assays was done with 180 unselected blood samples from healthy donors and patients (27). For both multimer and IFN- $\gamma$ Elispot assays, the detection limit was 100 cells in $10^{6} \mathrm{CD}^{+} \mathrm{T}$ cells $(0.01 \%)$ (42). Repeated analyses showed $15 \% \pm 16 \%$ (mean $\pm \mathrm{SD}$ ) variation in multimer ${ }^{+}$cells $(37)$. Good reproducibility was also found for the Elispot assay (variation of $30 \% \pm 21 \%$ ). In addition, longitudinal intraindividual result variability was assessed by testing multiple blood samples from individuals without a $\mathrm{T}$ cell response against Melan-A. Variation coefficients (SD in percent of mean multimer ${ }^{+}$values) were $20 \% \pm 27 \%$. For IFN- $\gamma$ Elispot assays, negative controls with all PBMC samples had a mean of less than $0.003 \%$ spots among $\mathrm{CD}^{+} \mathrm{T}$ cells, confirming that the background was far below the detection limit of $0.01 \%$.

Cell sorting, cDNA amplification, and 5-cell RT-PCR. CD8 ${ }^{+} \mathrm{T}$ cells were enriched with a MiniMACS device and stained with multimers and antibodies as described above. Five-cell aliquots were sorted directly with a FACSVantage SE machine into wells of 96-V bottom plates. cDNA preparation, $\mathrm{cDNA}$ amplification, and PCR were done using primers for CD3, granzyme B, perforin, IFN- $\gamma$,
TNF- $\gamma$, and CD94 as described (33). Either water or Daudi B cell line extract was used as a negative PCR control (-); $10^{3}$ PBMCs from a healthy individual were used as a positive control $(+)$.

$T$ cell cloning and cytotoxicity assay. Multimer ${ }^{+} \mathrm{CD}^{+} \mathrm{T}$ cells were sorted by flow cytometry, cloned by limiting dilution, and expanded with phytohemagglutinin (PHA) and allogenic feeder cells in medium containing $150 \mathrm{U} / \mathrm{ml}$ human recombinant IL-2 (hrIL-2) and $10 \mathrm{ng} / \mathrm{ml}$ hrIL-7. Subsequently, they were periodically (every 3-4 weeks) restimulated with PHA, irradiated feeder cells, and hrIL-2. Lytic activity and antigen recognition were assessed functionally in 4-hour ${ }^{51} \mathrm{Cr}$ release assays (42). Target cells were T2 cells $\left(\mathrm{A}^{+} /\right.$Melan- $\left.\mathrm{A}^{-}\right)$and the melanoma cell lines Me 275 ( $\mathrm{A} 2^{+} /$Melan$\mathrm{A}^{+}$) and NA8 (A2+/Melan- $\left.\mathrm{A}^{-}\right)$(31). The percentage of specific lysis was calculated as follows: $100 \times([$ experimental - spontaneous release $] /[$ total - spontaneous release]).

\section{Acknowledgments}

We thank the patients for study participation and blood donation, and the hospital staff for excellent collaboration. We are obliged to the Institutional Review Boards for suggestions and approvals. We gratefully acknowledge L.J. Old, H.F. Oettgen, and E.W. Hoffman for support; V. Appay, A. Baur, H. Davis, P.-Y. Dietrich, S. Efler, L. Guillou, C. Haarman, F.-A. Le Gal, S. Leyvraz, J.-A. Lobrinus, J.-Y. Meuwly, O. Michielin, L. Pan, C. Picht, M. Rafii, R. Venhaus, J. Whisnant, and A. Wolfer for collaboration and advice; R. Murphy for peptides; M. Lipp for antibodies; V. Aubert, K. Fleischhauer, B. Mazzi, and J.-M. Tiercy for HLA typing; P. Guillaume, I. Luescher, and the LICR Central Tetramer Facility for multimers; Seppic for Montanide ISA-51 (IFA); and Coley Pharmaceutical Group for CpG 7909. We also are grateful for the excellent technical and secretarial help of C. Baroffio, P. Corthesy, E. Devevre, C. Geldhof, R. Milesi, D. Minaidis, N. Montandon, K. Muehlethaler, M. van Overloop, S. Reynard, and S. Salvi. This study was sponsored and supported by the Ludwig Institute for Cancer Research, and by the National Center of Competence in Research (NCCR) Molecular Oncology, a research program of the Swiss National Science Foundation.

Received for publication September 16, 2004, and accepted in revised form December 7, 2004.

Address correspondence to: Daniel Speiser, Division of Clinical Onco-Immunology, Ludwig Institute for Cancer Research, Hôpital Orthopédique, Niveau 5 Est, Av. Pierre-Decker 4, CH-1005 Lausanne, Switzerland. Phone: 41-21-314-01-82; Fax: 41-21-314-74-77; E-mail: daniel.speiser@hospvd.ch.
1. Janeway, C.A., Jr. 1989. Approaching the asymptote? Evolution and revolution in immunology. Cold Spring Harb. Symp. Quant. Biol. 54:1-13.

2. Marrack, P., and Kappler, J. 1994. Subversion of the immune system by pathogens. Cell. 76:323-332.

3. Medzhitov, R., and Janeway, C.A., Jr. 1997. Innate immunity: impact on the adaptive immune response. Curr. Opin. Immunol. 9:4-9.

4. Akira, S., Takeda, K., and Kaisho, T. 2001. Tolllike receptors: critical proteins linking innate and acquired immunity. Nat. Immunol. 2:675-680.

5. Kadowaki, N., et al. 2001. Subsets of human dendritic cell precursors express different toll-like receptors and respond to different microbial antigens. J. Exp. Med. 194:863-869.

6. Steinman, R.M. 1991. The dendritic cell system and its role in immunogenicity. Annu. Rev. Immunol.
9:271-296.

7. Klinman, D.M. 2004. Immunotherapeutic uses of $\mathrm{CpG}$ oligodeoxynucleotides. Nat. Rev. Immunol. 4:249-259.

8. Hemmi, H., et al. 2000. A Toll-like receptor recognizes bacterial DNA. Nature. 408:740-745.

9. Takeshita, F., et al. 2001. Cutting edge: Role of Tolllike receptor 9 in CPG DNA-induced activation of human cells. J. Immunol. 167:3555-3558.

10. Weiner, G.J., Liu, H.M., Wooldridge, J.E., Dahle, C.E., and Krieg, A.M. 1997. Immunostimulatory oligodeoxynucleotides containing the $\mathrm{CPG}$ motif are effective as immune adjuvants in tumor antigen immunization. Proc. Natl. Acad. Sci. U. S. A. 94:10833-10837.

11. Lipford, G.B., et al. 1997. CpG-containing synthetic oligonucleotides promote B and cytotoxic T cell responses to protein antigen: a new class of vaccine adjuvants. Eur. J. Immunol. 27:2340-2344.

12. Oxenius, A., Martinic, M.M., Hengartner, H., and Klenerman, P. 1999. CpG-containing oligonucleotides are efficient adjuvants for induction of protective antiviral immune responses with T-cell peptide vaccines. J. Virol. 73:4120-4126.

13. Vabulas, R.M., Pircher, H., Lipford, G.B., Hacker, H., and Wagner, H. 2000. CpG-DNA activates in vivo $T$ cell epitope presenting dendritic cells to trigger protective antiviral cytotoxic $\mathrm{T}$ cell responses. J. Immunol. 164:2372-2378.

14. Davila, E., and Celis, E. 2000. Repeated administration of cytosine-phosphorothiolated guanine-containing oligonucleotides together with peptide/ protein immunization results in enhanced CTL responses with anti-tumor activity. J. Immunol. 
165:539-547.

15. Miconnet, I., et al. 2002. CpG are efficient adjuvants for specific CTL induction against tumor antigenderived peptide. J. Immunol. 168:1212-1218.

16. Hartmann, G., et al. 2000. Delineation of a CpG phosphorothioate oligodeoxynucleotide for activating primate immune responses in vitro and in vivo. J. Immunol. 164:1617-1624.

17. Bauer, S., et al. 2001. Human TLR9 confers responsiveness to bacterial DNA via species-specific CpG motif recognition. Proc. Natl. Acad. Sci. U. S. A. 98:9237-9242.

18. Krieg, A.M. 2002. CpG motifs in bacterial DNA and their immune effects. Annu. Rev. Immunol. 20:709-760.

19. Davis, H.L., et al. 2000. CpG DNA overcomes hyporesponsiveness to hepatitis $\mathrm{B}$ vaccine in orangutans. Vaccine. 18:1920-1924.

20. Halperin, S.A., et al. 2003. A phase I study of the safety and immunogenicity of recombinant hepatitis B surface antigen co-administered with an immunostimulatory phosphorothioate oligonucleotide adjuvant. Vaccine. 21:2461-2467.

21. Cormier, J.N., et al. 1997. Enhancement of cellular immunity in melanoma patients immunized with a peptide from MART-1/Melan A. Cancer J. Sci. Am. 3:37-44.

22. Pullarkat, V., et al. 2003. A phase I trial of SD-9427 (progenipoietin) with a multipeptide vaccine for resected metastatic melanoma. Clin. Cancer Res. 9:1301-1312

23. Liénard, D., et al. 2004. Ex vivo detectable activation of Melan-A specific T cells correlating with inflammatory skin reactions in melanoma patients vaccinated with peptides in IFA. Cancer Immunity. 4:4.

24. Rosenberg, S.A., et al. 1998. Immunologic and therapeutic evaluation of a synthetic peptide vaccine for the treatment of patients with metastatic melanoma. Nat. Med. 4:321-327.

25. Schaed, S.G., et al. 2002. T-cell responses against tyrosinase $368-376(370 \mathrm{D})$ peptide in HLA*A0201+ melanoma patients: randomized trial comparing incomplete Freund's adjuvant, granulocyte macrophage colony-stimulating factor, and QS-21 as immunological adjuvants. Clin Cancer Res. 8:967-972.

26. Speiser, D.E., et al. 2003. Disease-driven T cell acti- vation predicts immune responses to vaccination against melanoma. Cancer Immunity. 3:12.

27. Speiser, D.E., et al. 2004. Ex vivo analysis of human antigen specific CD8+ T cell responses: Quality assessment of fluorescent HLA-A2 multimers and IFN gamma Elispot assays for patient immune monitoring. J. Immunother. 27:298-308.

28. Kawabe, Y., and Ochi, A. 1991. Programmed cell death and extrathymic reduction of $\mathrm{V} \beta 8+\mathrm{CD} 4+$ $\mathrm{T}$ cells in mice tolerant to Staphylococcus aureus enterotoxin B. Nature. 349:245-248.

29. Lenardo, M., et al. 1999. Mature T lymphocyte apoptosis-immune regulation in a dynamic and unpredictable antigenic environment. Annu. Rev. Immunol. 17:221-253.

30. Blattman, J.N., et al. 2003. Therapeutic use of IL-2 to enhance antiviral T-cell responses in vivo. Nat. Med. 9:540-547.

31. Romero, P., et al. 2002. Antigenicity and immunogenicity of Melan-A/MART-1 derived peptides as targets for tumor reactive CTL in human melanoma. Immunol. Rev. 188:81-96.

32. Sallusto, F., Lenig, D., Forster, R., Lipp, M., and Lanzavecchia, A. 1999. Two subsets of memory T lymphocytes with distinct homing potentials and effector functions. Nature. 401:708-712.

33. Rufer, N., et al. 2003. Ex vivo characterization of human $\mathrm{CD} 8+\mathrm{T}$ subsets with distinct replicative history and partial effector functions. Blood. 102:1779-1787.

34. Boon, T., and Van den Eynde, B. 2003. Tumour immunology. Curr. Opin. Immunol. 15:129-130.

35. Monsurro, V., et al. 2001. Kinetics of TCR use in response to repeated epitope-specific immunization. J. Immunol. 166:5817-5825.

36. Jaeger, E., et al. 1996. Generation of cytotoxic T cell responses with synthetic melanoma associated peptides in vivo, implications for tumor vaccines with melanoma associated antigens. Int. J. Cancer. 66:162

37. Speiser, D.E., et al. 2003. Evaluation of melanoma vaccines with molecularly defined antigens by ex vivo monitoring of tumor specific T cells. Semin. Cancer Biol. 13:461-472.

38. Powell, D.J., Jr., and Rosenberg, S.A. 2004. Phenotypic and functional maturation of tumor antigen-reactive CD8+ $\mathrm{T}$ lymphocytes in patients undergoing multiple course peptide vaccination.
J. Immunother. 27:36-47.

39. Heikenwalder, M., et al. 2004. Lymphoid follicle destruction and immunosuppression after repeated CpG oligodeoxynucleotide administration. Nat. Med. 10:187-192.

40. Sparwasser, T., et al. 1999. Immunostimulatory CpG-oligodeoxynucleotides cause extramedullary murine hemopoiesis. J. Immunol. 162:2368-2374.

41. Salio, M., Palmowski, M.J., Atzberger, A., Hermans, I.F., and Cerundolo, V. 2004. CpG-matured murine plasmacytoid dendritic cells are capable of in vivo priming of functional CD8 $\mathrm{T}$ cell responses to endogenous but not exogenous antigens. J. Exp. Med. 199:567-579.

42. Pittet, M.J., et al. 1999. High frequencies of naive Melan-A/MART-1-specific CD8+ T cells in a large proportion of human histocompatibility leukocyte antigen (HLA)-A2 individuals. J. Exp. Med. 190:705-715

43. Yu, P., et al. 2004. Priming of naive T cells inside tumors leads to eradication of established tumors. Nat. Immunol. 5:141-149.

44. Houghton, A.N. 2004. LIGHTing the way for tumor immunity. Nat. Immunol. 5:123-124.

45. Verthelyi, D., Ishii, K.J., Gursel, M., Takeshita, F., and Klinman, D.M. 2001. Human peripheral blood cells differentially recognize and respond to two distinct CPG motifs. J. Immunol. 166:2372-2377.

46. Hartmann, G., et al. 2003. Rational design of new CPG oligonucleotides that combine B cell activation with high IFN-alpha induction in plasmacytoid dendritic cells. Eur. J. Immunol. 33:1633-1641.

47. Marshall, J.D., et al. 2003. Identification of a novel CpG DNA class and motif that optimally stimulate $B$ cell and plasmacytoid dendritic cell functions. J. Leukoc. Biol. 73:781-792.

48. Vollmer, J., et al. 2004. Characterization of three CPG oligodeoxynucleotide classes with distinct immunostimulatory activities. Eur. J. Immunol. 34:251-262.

49. Akbar, A.N., Lord, J.M., and Salmon, M. 2000. IFNalpha and IFN-beta: a link between immune memory and chronic inflammation. Immunol. Today. 21:337-342.

50. Sprent, J., and Surh, C.D. 2002. T cell memory. Annu. Rev. Immunol. 20:551-579.

51. Altman, J.D., et al. 1996. Phenotypic analysis of antigen-specific T lymphocytes. Science. 274:94-96. 